\title{
Synthesis and Quantum-Chemical Studies of New Hybrid Heterocyclic Molecules Derived from 3-(Thiocyanatoacetyl)Coumarin ${ }^{\dagger}$
}

\author{
Natalya S. Tatarchenko ${ }^{1}$, Vyacheslav K. Kindop ${ }^{1}$, Alexander V. Bespalov ${ }^{1}$, Victor V. Dotsenko ${ }^{1,2,3, *}$ and \\ Nicolai A. Aksenov ${ }^{3}$ \\ 1 Department of Organic Chemistry and Technologies, Kuban State University, 149 Stavropolskaya str, \\ 350040 Krasnodar, Russia; tat_natasha@mail.ru (N.S.T.); slavakindop@mail.ru (V.K.K.); \\ bespalov-alex@mail.ru (A.V.B.) \\ 2 ChemEx Lab, Vladimir Dal' Lugansk National University, 20A/7 Molodezhny, 91034 Lugansk, Russia \\ 3 Department of Chemistry, North Caucasus Federal University, 1a Pushkin St., 355009 Stavropol, Russia; \\ radioanimation@rambler.ru \\ * Correspondence: victor_dotsenko_@mail.ru \\ + Presented at the 24th International Electronic Conference on Synthetic Organic Chemistry, \\ 15 November-15 December 2020; Available online: https://ecsoc-24.sciforum.net/.
}

Citation: Tatarchenko, N.S.; Kindop, V.K.; Bespalov, A.V.; Dotsenko, V.V.; Aksenov, N.A. Synthesis and Quantum-Chemical Studies of New Hybrid Heterocyclic Molecules Derived from 3-(Thiocyanatoacetyl) Coumarin. 2021, 3, 78. https:// doi.org/10.3390/ecsoc-24-08104

Academic Editors: Julio A. Seijas and M. Pilar Vázquez-Tato

Published: 13 November 2020

Publisher's Note: MDPI stays neutral with regard to jurisdictional claims in published maps and institutional affiliations.

Copyright: (C) 2020 by the authors. Licensee MDPI, Basel, Switzerland. This article is an open access article distributed under the terms and conditions of the Creative Commons Attribution (CC BY) license (http://creativecommons.org/licenses /by/4.0/).
Abstract: 3-(Thiocyanatoacetyl)coumarin, prepared by a reaction of 3-(bromoacetyl)coumarin with $\mathrm{KSCN}$, reacts with primary aryl amines to give new hybrid heterocyclic products, 3-(3-aryl-2imino-2,3-dihydrothiazol-4-yl)-2H-chromen-2-ones. The structures and conformational features of the products have been studied by the B3LYP-D3/6-311G(d,p) method.

Keywords: 3-(bromoacetyl)coumarin; 3-(thiocyanatoacetyl)coumarin; heterocyclization; thiazolidines; DFT calculations

\section{Introduction}

Coumarins belong to privileged scaffolds useful as a starting point for preparation of drug-like molecules [1]. We focused our interests on 3-thiocyanatoacetylcoumarins 1, which are readily available through the bromination of 3-acetylcoumarins followed by subsequent nucleophilic substitution of the bromine atom with the $\mathrm{S}-\mathrm{C} \equiv \mathrm{N}$ group [2-4]. Such thiocyanates have a high reactivity and are useful as reagents for preparation of polyfunctional and poly-heterocyclic structures. In general, $\alpha$-thiocyanatocarbonyl compounds can be used in the synthesis of sulfur-containing heterocycles with a potential biological activity (for reviews, see References [5-7]).

\section{Results and Discussion}

Thus, the reaction of compound $\mathbf{1}$ with anilines in an acidic medium lead to the formation of hybrid polycyclic systems 2 bearing the iminothiazoline fragment (Scheme 1). Such coumarin derivatives are of practical interest as perspective candidates for bio-screening [8-11]. 


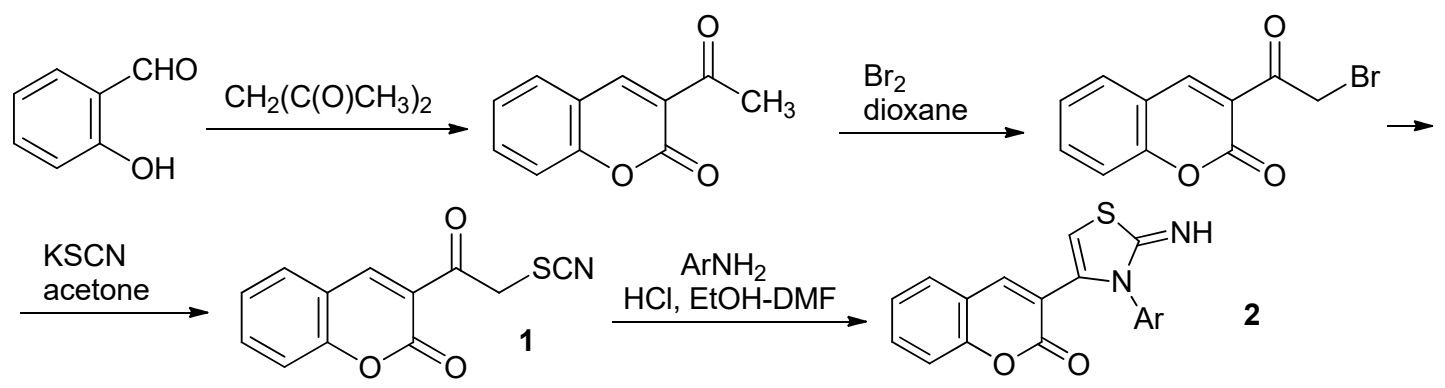

Scheme 1. The synthetic pathway to hybrid molecules 2 .

The presence of the imino group in the thiazole fragment provides very wide synthetic possibilities. Thus, the reaction of 2 with chloroacetyl chloride proceeds smoothly to give new chloroacetamides 3 (Scheme 2) useful as alkylating agents and capable of introducing hybrid polycyclic fragments into molecules.

The resulting compounds 2 were characterized using spectral methods (IR, NMR spectroscopy). In addition, quantum-chemical DFT (density functional theory) calculations of the most stable conformers of compound $\mathbf{2 a}(\mathrm{R}=\mathrm{H})$ were performed (Figure 1).<smiles>N=c1scc(-c2cc3ccccc3oc2=O)n1[Ga]</smiles><smiles>O=C(CCl)/N=c1/scc(-c2cc3ccccc3oc2=O)n1[Al]</smiles>

Scheme 2. The acylation of iminothiazolines 2.

The preliminary conformational analysis of 3-(2-imino-3-phenyl-2,3-dihydrothiazol4-yl)-2H-chromen-2-one using the semi-empirical RM1 method showed that this molecule can exist as two conformers (s-trans and s-cis), which are due to rotation around the C8-C11 bond (Figure 1). Since semiempirical methods in many cases led to incorrect results, further geometrical optimization and energy DFT calculations were performed using the well-known functional B3LYP. The D3 Grimme's correction was used to describe the dispersion contribution to the energy to allow one to discuss the effect of the Van der Waals forces. The choice of the split valence basis set 6-311(d, p) was due to a compromise between the accuracy and the time of the calculation. The molecular structures of these conformers that were optimized using the B3LYP-D3/6-311G (d, p) method are shown in Figure 1. According to the calculated data, the difference in energy between these conformations is $5.5 \mathrm{~kJ} / \mathrm{mol}$ (the s-trans conformer is more stable). The C7-C8-C11-N12 torsion angle between the coumarin and thiazole fragments in the s-trans conformer is $24^{\circ}$, while the analogous C9-C8-C11-N12 angle in the s-cis conformer is $56^{\circ}$. It clearly points to the absence of conjugation between the heterocyclic cores. The C11-N12-C16-C21 torsion angle, which characterizes the mutual arrangement of the thiazole ring and aromatic ring in these conformers has somewhat closer values $\left(45^{\circ}\right.$ and $55^{\circ}$, respectively). 

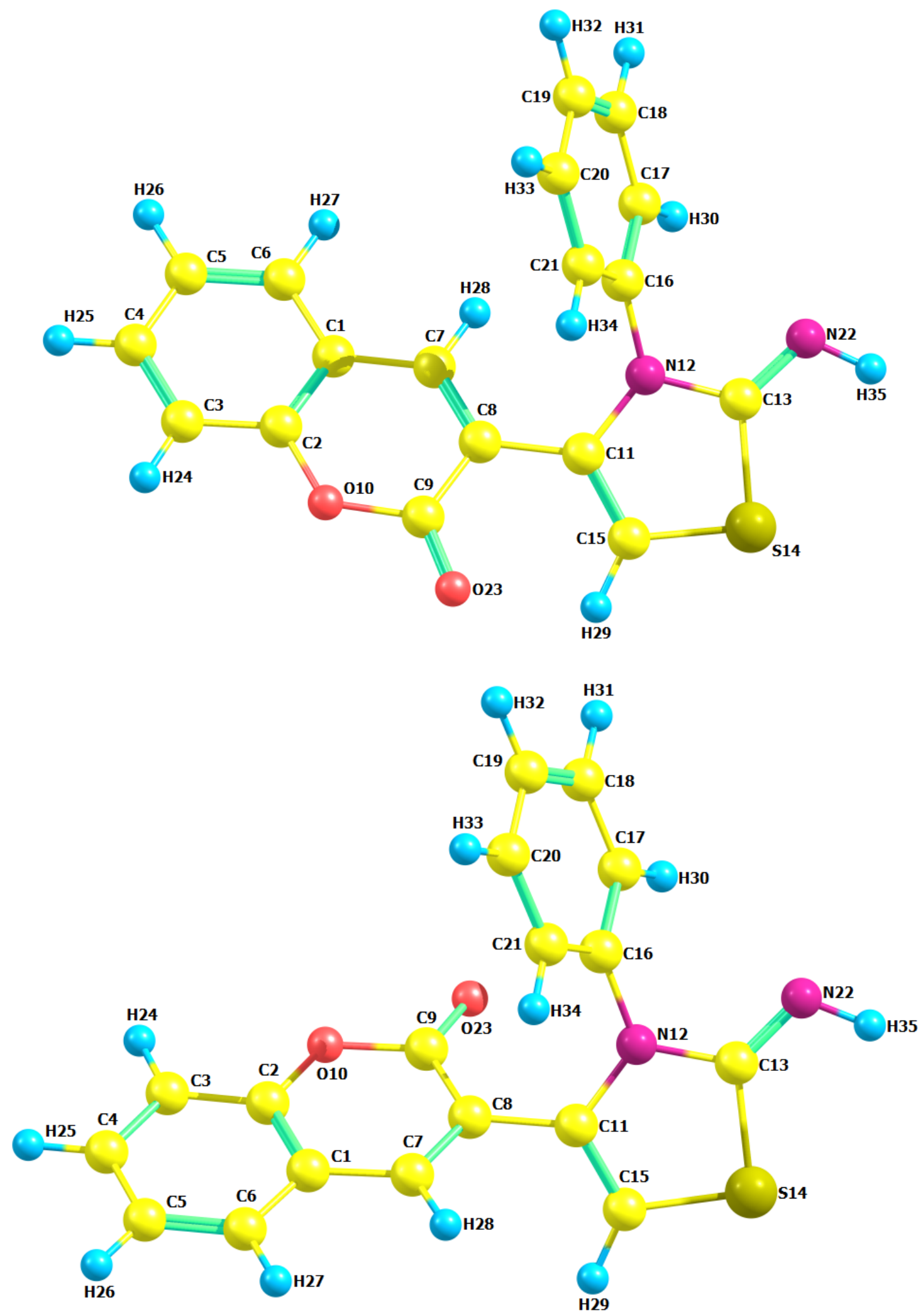

Figure 1. The structure of s-trans (upper) and s-cis (lower) conformers of 3-(2-imino-3-phenyl-2,3-dihydrothiazol4-yl)-2H-chromen-2-one according to quantum-chemical studies. 


\section{Experimental}

\subsection{3-(Thiocyanatoacetyl)coumarin (1).}

3-Bromoacetylcoumarin (11.25 g, $0.05 \mathrm{~mol})$ was placed in a flask and dissolved in $20 \mathrm{~mL}$ of acetone upon gentle heating. In the resulting solution, $5.8 \mathrm{~g}(0.06 \mathrm{~mol})$ of potassium thiocyanate was added slowly with stirring. The formation of white precipitate $(\mathrm{KBr})$ was observed. After $2 \mathrm{~h}$, the reaction mixture was diluted with cold water, and the precipitated light-yellow solid was filtered off and recrystallized from acetone- $\mathrm{EtOH}$. The yield was $7.7 \mathrm{~g}(67 \%)$. The purity of the product was checked by TLC (Sorbfil plates, "Imid Ltd." (Krasnodar)) using acetone: $\mathrm{CCl}_{4} 1: 1$ mixture as an eluent.

IR spectrum $\left(v, \mathrm{~cm}^{-1}\right): 1708,1673(\mathrm{C}=\mathrm{O}), 1598(\mathrm{C}=\mathrm{C}), 2156(\mathrm{SCN})$ (Figure 2).

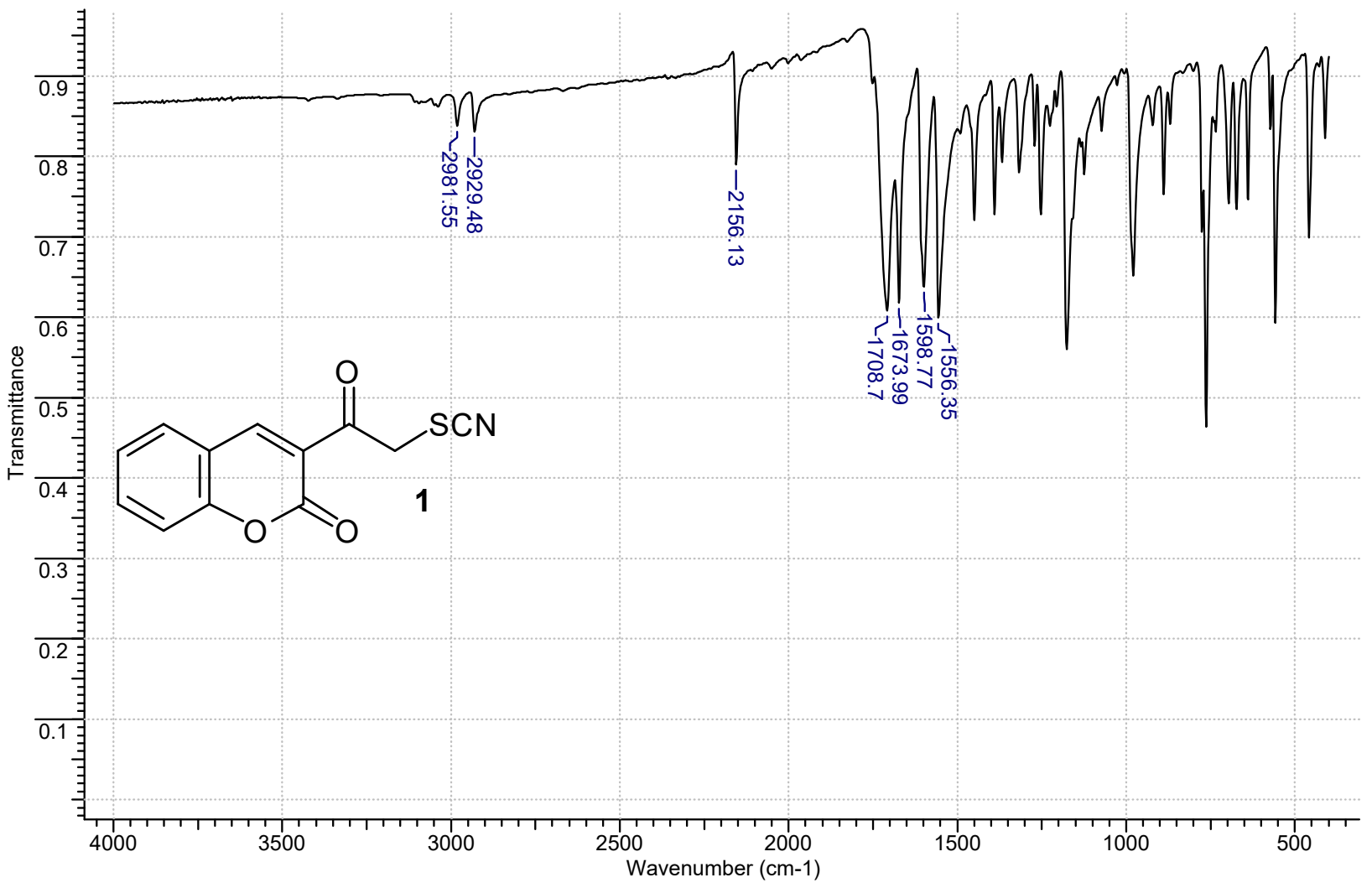

Figure 2. ATR-FT-IR spectrum of compound 1.

\subsection{Synthesis of 3-(2-imino-3-phenyl-2,3-dihydrothiazol-4-yl)-2H-chromen-2-one (2a)}

Aniline $(0.6 \mathrm{~mL}, 0.005 \mathrm{~mol})$ was placed in a flask and neutralized with an equivalent amount of concentrated aq. $\mathrm{HCl}$. The resulting hydrochloride was dissolved in $\mathrm{EtOH}$. To the solution of $\mathrm{PhNH}_{2} \times \mathrm{HCl}$, a solution of $1.0 \mathrm{~g}(0.005 \mathrm{~mol})$ of thiocyanatoacetylcoumarin (1) in a minimum volume of 1:1 DMF-EtOH mixture was added. A gaseous $\mathrm{HCl}$ was passed through the reaction mixture for $2 \mathrm{~h}$. Then, the mixture was diluted with water to give a light-yellow precipitate. The yield of product (2a) was $1.0 \mathrm{~g}(64 \%)$. The purity of the product was checked by TLC (thin-layer chromatography) (Sorbfil plates, "Imid Ltd." (Krasnodar, Russia) using acetone: $\mathrm{CCl}_{4}$ 1:1 mixture as an eluent. IR spectrum $\left(v, \mathrm{~cm}^{-1}\right)$ : $3029(\mathrm{~N}-\mathrm{H}), 1724(\mathrm{C}=\mathrm{O}), 1604(\mathrm{C}=\mathrm{N})$.

Author Contributions: Conceptualization, V.V.D., A.V.B.; methodology, V.V.D., A.V.B.; synthesis and analysis, N.S.T., V.K.K., N.A.A.; calculations: V.K.K., A.V.B.; writing-original draft preparation, V.V.D., A.V.B.; writing - review and editing, V.V.D.; supervision, V.V.D.; funding acquisition, V.V.D. All authors have read and agreed to the published version of the manuscript.

Institutional Review Board Statement: Not applicable. 
Informed Consent Statement: Not applicable.

Data Availability Statement: Not applicable.

Funding: The reported study was funded by RFBR and Krasnodar region according to the research project № 19-43-230007.

Conflicts of Interest: The authors declare no conflict of interest.

\section{References}

1. $\quad$ Borges, M.F.M.; Roleira, F.M.F.; Milhazes, N.J.S.P.; Villare, E.U.; Penin, L.S. Simple coumarins: Privileged scaffolds in medicinal chemistry. Front. Med. Chem. 2010, 4, 23-85.

2. Yagodinets, P.I.; Skripskaya, O.V.; Prodanchuk, N.G.; Chernyuk, I.N.; Sinchenko, V.G.; Dozirtsiv, G.M.; Pityk, M.Y. Synthesis and antimicrobial activity of derivatives of phosphorus-and nitrogen-containing coumarins. Pharm. Chem. J. 1995, 29, 54-56.

3. Ramanna, S.; Rao, V.R.; Kumari, T.S.; Padmanabha Rao, T.V. Synthesis of N-(4-2H-1-benzopyran-2-one- 2-thiazolyl)phthalimides. Phosphorus Sulfur Silicon Relat. Elem. 1995, 107, 197-204.

4. Rao, V.R.; Reddy, V.R. A facile synthesis of some new 3-(2-hydroxy-4-thiazolyl) coumarins and their derivatives. Heterocycl. Commun. 2003, 9, 635-640.

5. Castanheiro, T.; Suffert, J.; Donnard, M.; Gulea, M. Recent advances in the chemistry of organic thiocyanates. Chem. Soc. Rev. 2016, 45, 494-505.

6. Gouda, M.A. 1-Phenyl-2-thiocyanatoethanone as Synthons in Heterocyclic Synthesis. Synth. Commun. 2013, 43, $2547-2574$.

7. Vekariya, R.H.; Patel, H.D. $\alpha$-Thiocyanation of carbonyl compounds: A review. Synth. Commun. 2017, 47, 87-104.

8. Arshad, A.; Osman, H.; Bagley, M.C.; Lam, C.K.; Mohamad, S.; Zahariluddin, A.S.M. Synthesis and antimicrobial properties of some new thiazolyl coumarin derivatives. Eur. J. Med. Chem. 2011, 46, 3788-3794.

9. Heravi, M.M.; Khaghaninejad, S.; Mostofi, M. Pechmann reaction in the synthesis of coumarin derivatives. Adv. Heterocycl. Chem. 2014, 112, 1-50.

10. Gunnewegh, E.A.; Hoefnagel, A.J.; Downing, R.S.; Van Bekkum, H. Environmentally friendly synthesis of coumarin derivatives employing heterogeneous catalysis. Recueil des Travaux Chimiques des Pays-Bas 1996, 115, $226-230$.

11. Medina, F.G.; Marrero, J.G.; Macías-Alonso, M.; González, M.C.; Córdova-Guerrero, I.; García, A.G.T.; Osegueda-Robles, S. Coumarin heterocyclic derivatives: Chemical synthesis and biological activity. Nat. Prod. Rep. 2015, 32, $1472-1507$. 